\title{
Solid Acid Pretreatment Enhancing the Production of VFAs from Corn Cob
}

\author{
Lele Liu, Jianwen Wei" \\ College of Environmental Science and Engineering, Guilin University of Technology, Guilin, China
}

Email address:

zhhk1202@163.com (Lele Liu), jianwen988@126.com (Jianwen Wei)

${ }^{*}$ Corresponding author

\section{To cite this article:}

Lele Liu, Jianwen Wei. Solid Acid Pretreatment Enhancing the Production of VFAs from Corn Cob. Science Discovery. Vol. 8, No. 6, 2020, pp. 122-127. doi: 10.11648/j.sd.20200806.11

Received: September 5, 2020; Accepted: September 23, 2020; Published: October 21, 2020

\begin{abstract}
Volatile fatty acids (VFAs) are products with high added value and widely used. The production of organic acids by anaerobic fermentation of biomass is an environmentally friendly method and can realize the recycling of waste. In order to solve the problem that biomass structure is complex and difficult to hydrolyze, three pretreatment methods, solid acid, dilute sulfuric acid and hydrothermal treatment, were used to pretreat corncob. The effects of three pretreatment methods on acid production of corncob by anaerobic fermentation were compared. The results showed that after 16 days of fermentation, the concentration of VFAs of solid acid pretreatment group was significantly higher than that of other experimental groups, reaching $4055.15 \mathrm{mg} / \mathrm{L}$, which was $41.34 \%$ higher than that of control group. The results showed that solid acid pretreatment can effectively destroy the structure of corncob, promoted its hydrolysis and increase the acid production of anaerobic fermentation. Solid acid pretreatment with the advantages of environmentally friendly, high efficiency and low cost is a promising process for biomass acid production, which promotes the production of VFAs by biomass fermentation.
\end{abstract}

Keywords: Solid Acid, Corn Cob, Volatile Fatty Acids (VFAs)

\section{固体酸预处理促进玉米芯生产挥发性脂肪酸}

\section{刘乐乐, 魏建文*}

桂林理工大学环境科学与工程学院, 广西桂林, 中国

\section{邮箱}

zhhk1202@163.com(刘乐乐), jianwen988@126.com(魏建文)

摘要：挥发性脂肪酸（volatile fatty acids, VFAs）是一类具有高附加值和应用广泛的产品。利用生物质庈氧发酵生产有 机酸是一种环境友好并且可实现废物资源化的方法。本文针对生物质结构复杂难以水解的问题, 采用固体酸、稀硫酸 和水热三种预处理方法对玉米芯进行预处理, 比较了三种预处理方法对玉米芯厌氧发酵产酸的影响。结果表明, 发酵 $16 \mathrm{~d}$ 后, 固体酸预处理组VFAs浓度显著高于其他实验组, 可达 $4055.15 \mathrm{mg} / \mathrm{L}$, 相对于对照组提高了 $41.34 \%$ 。说明固体 酸预处理可以有效地破坏玉米芯结构促进其水解以至于提高厌氧发酵产酸量。固体酸预处理促进生物质发酵产VFAs 具有环境友好、高效、低廉的优点, 因此是一种具有前景的可推广的生物质预处理工艺。

关键词：固体酸, 玉米芯, 挥发性脂肪酸 


\section{1. 引言}

挥发性脂肪酸（volatile fatty acids, VFAs）作为重要 的有机碳源，可应用于生产生物柴油、生物甲烷和生物氢 气, 也可作为原料生产可生物降解塑料缓解白色污染问题, 具有较高的经济价值。但是目前我国生产VFAs主要采用 化学方法, 这会消耗大量的石化资源, 同时会造成环境污 染, 导致经济成本较大。最近很多研究表明, 通过生物质 (如剩余污泥、餐㕑和农林废弃物等) 厌氧发酵产VFAs 是一种经济、环保、高效的方法。Zhou等 (2014) 以污水 处理厂废弃活性污泥为底物, 蘑菇渣为碳水化合物添加剂 厌氧发酵产VFAs, 得到的最大酸产量为 $712 \pm 49$ $\mathrm{mgCOD} / \mathrm{gVSS}$, 比空白组提高了 $50 \%[1]$ 。Bahriye等 $(2020)$ 利用柑橘废料庈氧发酵产VFAs, 最高产量达到了 $0.793 \mathrm{~g}$ $\mathrm{VFA} / \mathrm{g}$ VS[2]。

我国作为农业大国, 每年都会有大量的农业废物, 这 为利用生物质发展生物质能源提供了重要的物质基础[3]。 但是木质纤维素生物质原料具有较高的聚合度和结晶度, 结构致密, 这会降低纤维素和半纤维素的水解效果和生物 能源的转化效率。已有研究表明, 通过预处理技术能够加 速破坏植物木质素、纤维素及半纤维素间复杂的空间结构, 降低聚合度, 促进水解反应的进行, 提高转化效率。因此, 预处理技术成为了充分利用木质纤维素生物质发展生物 质能源的关键技术。目前已经有很多预处理方法应用到生 物质原料产VFAs中，例如机械粉碎、水热预处理、酸和 碱预处理等。徐超等 (2011) 采用强碱对水生植物香蒲枯 叶进行预处理, 厌氧发酵产VFAs的量提高到了196.57 $\mathrm{mg} / \mathrm{g}$, 是没有经过预处理的2.2倍[4]。汪楚乔 (2017)发现 经过微氧预处理后油菜厌氧发酵产VFAs的量最高达到 $9072.1 \mathrm{mg} / \mathrm{L}$ ，相对于没有预处理的油菜提高了 $88.43 \%$ [5]。

酸预处理在预处理技术中广为研究和应用, 因为酸预 处理具有较高的预处理效果, 在工业过程中常常将无机酸 (如硫酸、盐酸等) 和有机酸 (如乙酸、草酸等) 应用于 酸处理。但是在使用液体酸的过程中, 经常会出现腐蚀设 备、溶于液相的酸难分离回收、产生的废液需要中和处理 等缺点 $[6]$ 。而固体酸与液体酸的预处理反应原理一致, 且 具有易储存、腐蚀性小、产物易分离、产生废物少、易回 收以及对环境无污染等优点[7]。因此, 以固体酸代替传统 液体酸具有重要的研究价值和意义。目前关于固体酸预处 理木质纤维素生物质厌氧发酵产VFAs的研究很少。本文研 究了固体酸和液体酸预处理对玉米芯厌氧发酵产酸的影响, 通过测定发酵系统中VFAs、SCOD、多糖和蛋白质浓度随 发酵时间的变化情况，分析不同预处理方法的厌氧产酸效 果, 比较固体酸和液体酸对玉米芯发酵产酸的促进效果。

\section{2. 实验材料和方法}

\section{1. 实验材料}

\subsection{1. 农业废物材料}

本实验采用的农业废料主要是稻壳和玉米芯。稻壳购 于浙江杭州, 在本实验中作为制备固体酸的原料。玉米芯
来自河北省保定市，作为水解产糖及厌氧发酵产VFAs的 底物。稻壳和玉米芯在室温下自然干燥 2 周, 干燥后用植 物粉碎机粉碎, 过 $40 \sim 60$ 目的篮子, 然后在烘箱中 $60^{\circ} \mathrm{C}$ 干 燥 $12 \mathrm{~h}$, 干燥后用自封袋密封, 做好便签并在室温下保存 以便后续使用。

\subsection{2. 接种污泥}

本实验所用的厌氧消化接种污泥购于安徽合肥碧达环 保科技有限公司。为了竟可能的去除产甲烷菌对发酵产生 的VFAs的消耗, 污泥在使用前先用破碎机进行破碎, 然后 在马弗炉上低温煮沸 $30 \mathrm{~min}$, 去除产甲烷菌的干扰, 最后 置于 $4^{\circ} \mathrm{C}$ 冰箱内保存备用。接种污泥的总固体TS和挥发性固 体VS分别为 $80133.31 \pm 967.12$ 和 $63409.67 \pm 712.56 \mathrm{mg} / \mathrm{L}$ 。

\section{2. 实验方法}

\subsection{1. 固体酸的制备}

制备固体酸主要有两种方法, 即“一步碳化-磺化法” 和“两步碳化-磺化法”。本研究采用“一步碳化-磺化法”制 备固体酸 [8]。“一步碳化-磺化法”即是指在反应过程中碳 原料的碳化、磺化在一个反应釜内一次完成, 即在反应釜 内同时加入稻壳和浓硫酸。稻壳在浓硫酸的脱水性和强酸 性的作用下脱水碳化, 反应后碳材料会变成多环芳烃结构 的材料, 磺酸基团接到杂化轨道上。

本研究采用“一步碳化-磺化法”制备固体酸, 这种制备 方式具有操作相对简单、能耗较少、反应速率快等优点。 先将处理好的稻壳放入水热合成反应器内, 在反应器内加 入 $90 \%$ 的浓硫酸, 边加边摚拌, 使原料与浓硫酸充分混合 而不结块。然后将水热反应釜密封, 放入 $100 \sim 200^{\circ} \mathrm{C}$ 的烘 箱中加热 $2 \sim 8 \mathrm{~h}$ 。水热合成后, 反应器自然冷却至室温, 在通风处将容器内的产物倒入冷水中, 边倒边搅拌, 洗涤 沉淀至少三次。然后用热水反复洗涤, 直至混合液 $\mathrm{pH}$ 呈中 性, 且检测不到 $\mathrm{SO}_{4}{ }^{2-}$ 离子。最后将收集的滤渣在 $105^{\circ} \mathrm{C}$ 的 烘箱中干燥 $24 \mathrm{~h}$, 在研钵中研磨至 200 目, 装入有标签的 自封袋中，室温下保存备用。

\subsection{2. 玉米芯预处理}

为比较不同预处理方法对玉米芯的发酵产酸的促进 效果, 分别采取固体酸、0.5\%稀硫酸和水热三种预处理方 式对玉米芯进行预处理。固体酸预处理是在反应釜中加入 $2 \mathrm{~g}$ 玉米芯和 $1 \mathrm{~g}$ 固体酸催化剂, 加蒸馏水至 $50 \mathrm{ml}$, 搅拌均 匀; 稀硫酸预处理和水热预处理则分别在 $50 \mathrm{ml}$ 的 $0.5 \%$ 稀 硫酸溶液和 $50 \mathrm{ml}$ 的热水中加入 $2 \mathrm{~g}$ 玉米芯。将密封的反应 釜置于 $100 \sim 160^{\circ} \mathrm{C}$ 的恒温箱中持续加热 $1 \mathrm{~h}$ 。反应完成后自 然冷却至室温使用抽滤方法将水解液与水解固体酸进行 固液分离。滤渣包括被处理过的玉米芯水解残渣和固体酸, 可根据它们的粒径差异, 通过筛分等操作, 分离出固体酸, 达到回收再使用的目的。

\subsection{3. 预处理后的玉米芯产VFAs}

将不同预处理后的玉米芯残渣及水解液分别加入 250 $\mathrm{ml}$ 的玻璃血清瓶中, 按照 VS 比为 $1: 1$ 加入接种污泥, 摇匀, 
调整pH至7, 加去离子水定容至 $200 \mathrm{ml}$ 。发酵瓶用塞子密 封, 并向血清瓶中充入 $2 \mathrm{~min}$ 氮气, 排出氧气，保持发酵 环境为厌氧环境。本实验设置一个对照组, 将 $2 \mathrm{~g}$ 未处理 的玉米芯加入血清瓶中, 加入同上等量的接种污泥, 加去 离子水定容至 $200 \mathrm{ml}$ 。将所有发酵瓶置于恒温水浴振荡器 中, 设置温度为 $37^{\circ} \mathrm{C}$, 震荡速度为 $100 \mathrm{rpm} / \mathrm{min}$ 。每组实验 设置 3 个平行, 计算结果为三个平行实验的平均值。每隔 一定时间取发酵液测定溶解性化学需氧量（SCOD）、蛋 白质、多糖、VFAs含量及其组分。

\section{3. 检测及分析方法}

\subsubsection{VFAs各组分浓度测定}

发酵液中VFAs各组分及其含量采用气相色谱法测定。 气相色谱仪 (岛津GC-2010 Pro) 使用的检测器为火焰离 子化检测器 (FID), 以氦气为载气, 氧气和氢气为燃气, 检测器和进样口温度均设置为 $250^{\circ} \mathrm{C}$ 。首先将发酵液在 $10000 \mathrm{r} / \mathrm{min}$ 的离心机下离心 $10 \mathrm{~min}$, 然后将上清液用 0.25 $\mu \mathrm{m}$ 膜过滤, 滤液与 $3 \%$ 的磷酸溶液以 $10: 1$ 注入进样品瓶混 合摇匀, 最后取 $1 \mathrm{ml}$ 样品置于自动进样器中测定VFAs。

\subsubsection{SCOD和蛋白质浓度测定}

SCOD使用连华科技水质分析仪测定。水溶性蛋白含 量采用比色法测定, 以牛血清蛋白制作标曲。

\subsection{3. 多糖浓度的测定}

发酵液中多糖含量采用葱酮比色法测定。向 $0.1 \mathrm{~g}$ 蒽酮 中加入 $100 \mathrm{ml} 80 \%$ 的浓硫酸, 边加边摚拌, 现配现用。取 $1 \mathrm{ml}$ 样品置于试管中, 缓慢注入 $4 \mathrm{ml}$ 葱酮溶液, 混匀后迅 速放入冷水浴中冷却 $10 \mathrm{~min}$, 然后在 $100^{\circ} \mathrm{C}$ 下煮沸 $10 \mathrm{~min}$, 流水冷却至室温, 在 $625 \mathrm{~nm}$ 处测定吸光度, 代入标曲计算 多糖浓度。

\section{3. 实验结果及分析}

\section{1. 产酸发酵过程中VFAs浓度和组分变化}

\subsubsection{VFAs浓度变化}

在厌氧发酵过程中，VFAs产量是评价厌氧发酵产酸 效果最主要的参数。图1显示水热、稀硫酸及固体酸预处 理对玉米芯厌氧发酵产VFAs的影响。由图可知, 在反应 初始阶段, 各个实验组中VFAs产量都有不同程度的增加。 这可能是因为预处理使发酵液中微生物可直接利用的可 溶性有机物增多, 加快了初始厌氧发酵速度[9]。在所有发 酵系统中, VFAs基本为两段式增长。在前 3 天, VFAs产 量迅速增加; 第3至14天, VFAs产量增加缓慢; 最终, 在 14天后VFAs产量基本趋于稳定。综上表明, 在厌氧发酵 前期, 预处理后的玉米芯发酵产酸速度快, 这可能是由于 预处理后实现了玉米芯半纤维素和木质素的有效去除, 解 除了纤维素水解的主要障碍, 增加了水解产物的产量, 为 微生物提供了易利用的营养物质, 从而实现最高的VFAs 产量[10]。反应 $16 \mathrm{~d}$ 后, 固体酸预处理后的玉米芯厌氧产 酸量最高, 达到 $4055.15 \mathrm{mg} / \mathrm{L}$, 其次是稀硫酸预处理组,
达到 $2869.87 \mathrm{mg} / \mathrm{L}$, 分别比对照组产酸量提高 $41.34 \%$ 和 $26.45 \%$ 。水热预处理组发酵液中VFAs浓度为 $3005.24 \mathrm{mg} / \mathrm{L}$ 。 固体酸预处理后的底物厌氧发酵产酸量显著增加, 表明固 体酸预处理木质纤维素生物质是一种有效可行的预处理 方法, 并且固体酸在实验过程可以循环利用, 减少废物的 产生, 对环境无污染, 符合绿色可持续发展。综上所述, 固体酸可以作为催化剂强化玉米芯厌氧发酵产酸。

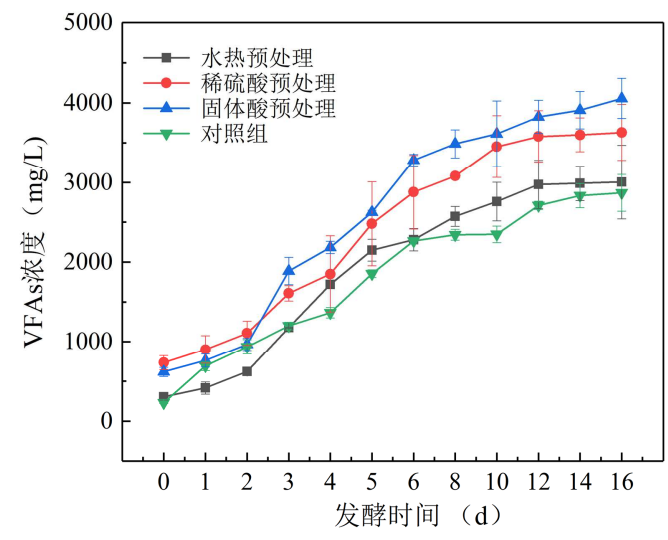

图1 不同预处理发酵液中VFAs浓度的变化。

\subsubsection{VFAs组分分布及变化}

VFAs的应用不仅与其产量有关，还与其组成成分有 关, 比如当VFAs作为外加碳源用于污水处理时, VFAs组 分和其比例将会影响微生物在废水处理过程中的活性, 进 而影响处理效果[11]。不同预处理方式对玉米芯厌氧发酵 产酸量及各组分质量浓度的影响如图2。从图中可以看出, 不同实验组中VFAs的组成成分及其比例相似, 主要为五 种: 乙酸、丁酸、异丁酸和异戊酸[12]。显然, 乙酸是所 有组最主要的VFAs, 含量占总产酸量的 $60 \%$ 以上。乙酸是 所有VFAs中最主要的成分, 可用于生产氢气、电力和沼 气, 也可以用来去除废水处理中的生物养分[13-14]。结果 表明, 玉米芯具有较高的乙酸生产潜能。其次是丁酸含量, 丁酸可以用来制备精细化工产品, 如香精、食品添加剂等 [15-16]。丙酸、异丁酸和异戊酸浓度都相对较低。三个预 处理组都不同程度地提高了乙酸和丁酸的含量, 这表示预 处理方式能够使玉米芯厌氧发酵产酸含量增加, 但对 VFAs的组成没有明显影响。

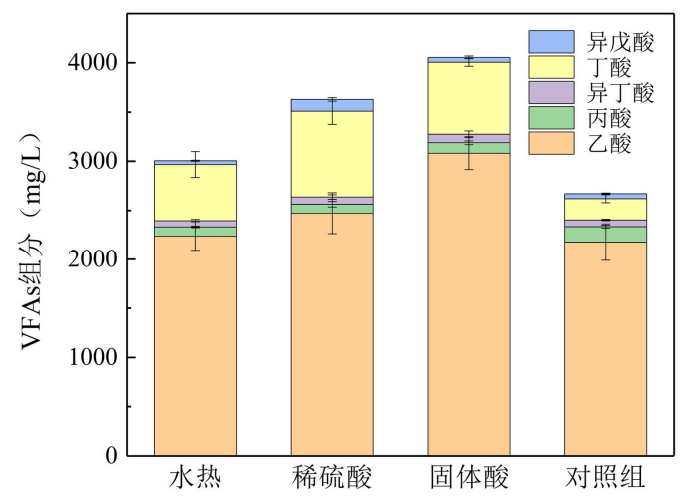

图2 不同预处理发酵液中VFAs的组成成分。 
在发酵产酸过程中，不同实验组发酵液的VFAs组分 浓度随时间的变化如图3所示。在厌氧发酵过程中, 乙酸 浓度一直最高, 而且除了稀硫酸预处理组, 其他实验组的 乙酸含量都随着时间的延长而增加, 说明乙酸的生成伴随 着整个发酵过程。随着反应的进行, 丁酸浓度相对提高, 并且在前 4 天逐渐增加, 4 天后丁酸含量基本保持稳定。所 有系统中的丙酸含量少量增加, 但依旧偏低。异丁酸和异 戊酸浓度一直较低, 并且没有明显的变化。四组实验产物 均主要为乙酸和丁酸, 表明厌氧发酵类型都是乙酸型发酵, 这与接种污泥的菌群有关。
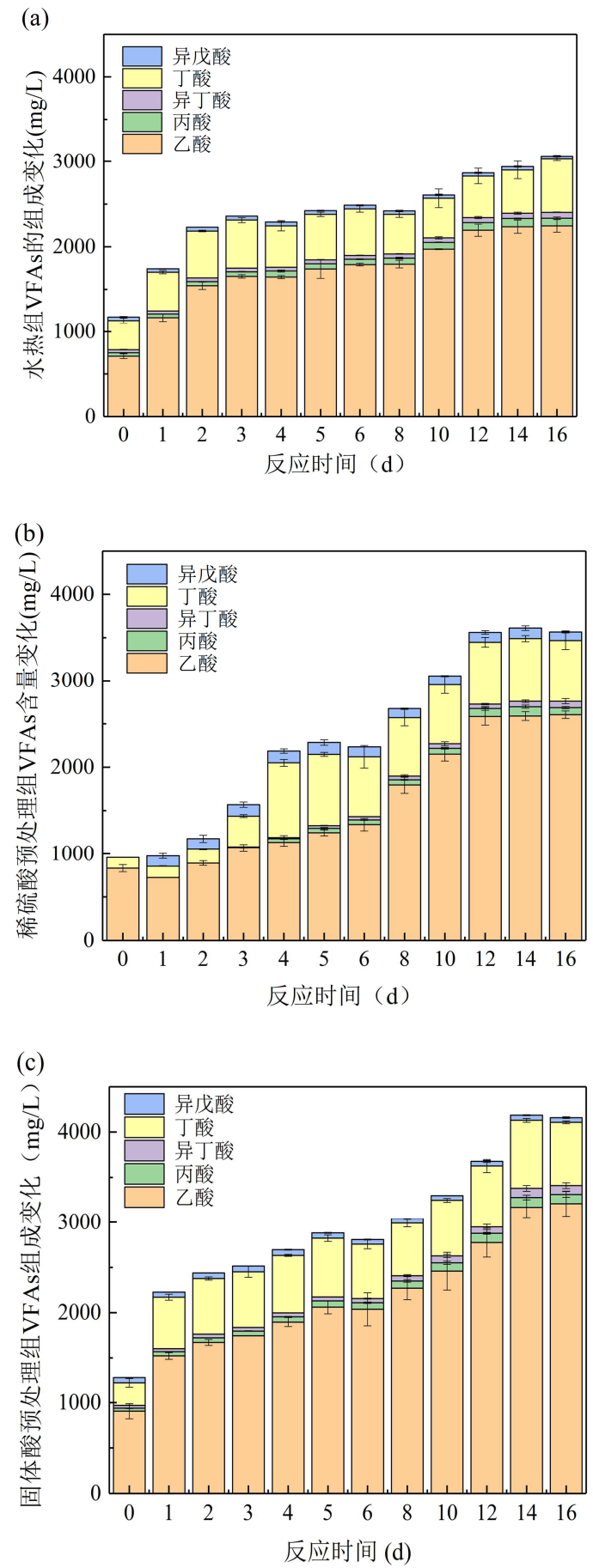

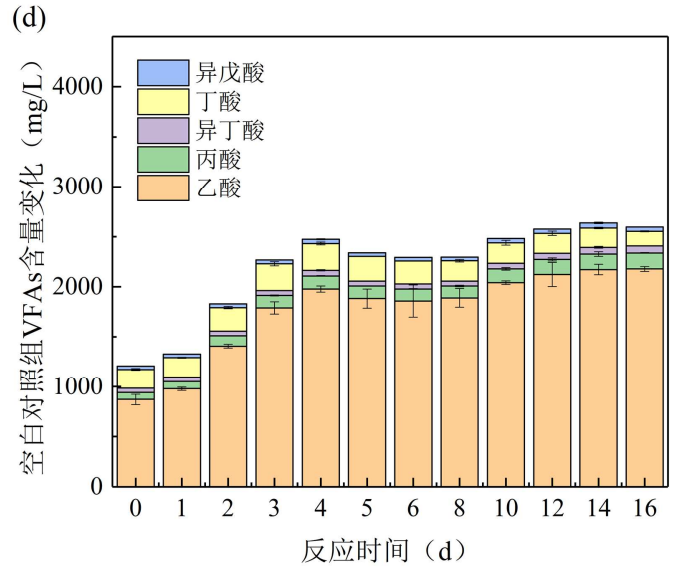

图3 不同预处理组发酵液中VFAs组成成分随时间的变化。

（a）水热预处理组（b）稀硫酸预处理组（c）固体酸预处理组（d）空 白对照组

\section{2. 发酵液中溶解性有机物浓度分析}

\subsubsection{SCOD变化}

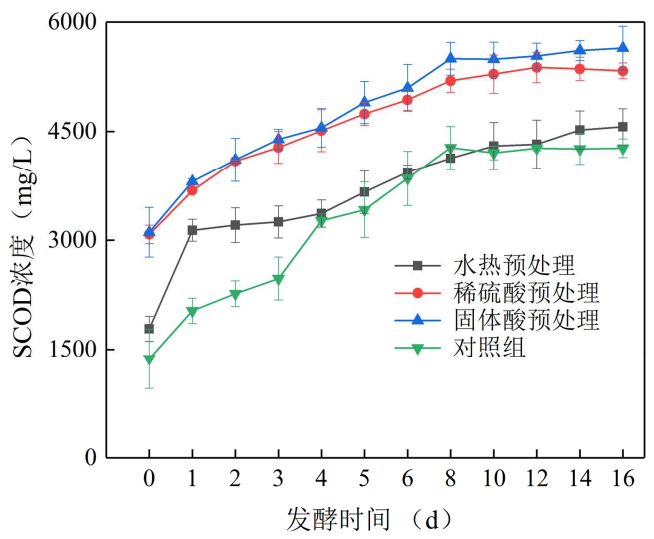

图4 发酵液中SCOD浓度的变化。

在水解和酸发酵系统中, 底物的降解能力可以通过 SCOD的浓度来表示[17-18]。SCOD浓度越高, 表明系统 释放了越多的有机物到水解酸化也中并为厌氧产酸发酵 提供了底物, 从而有利于VFAs的积累[11]。在发酵产酸过 程中, 不同实验组发酵液的SCOD浓度随时间的变化如图4 所示。所有系统中SCOD浓度的增长基本可以分为 $0-4$ 天和 5-10天两个阶段, 并且在 10 天左右达到最大。固体酸和稀 硫酸预处理组的玉米芯发酵液中总体SCOD浓度明显高于 水热预处理组和对照组, 并且在反应开始初期, 两种酸预 处理组的底物SCOD溶出量也高于水热预处理组和对照组。 随着反应的进行, 各实验组发酵液中SCOD浓度均呈波动 上升的趋势, 这主要源于玉米芯中的有机物在水解和微生 物的共同作用下继续溶解。SCOD浓度的变化能够反映固 体生物质向可溶性有机物的转化, 在本实验中, SCOD浓 度的变化反映的是玉米芯水解成可溶性有机物, 从 SCOD 浓度的变化可以看出, 固体酸预处理能够使玉米芯更容易 被水解产生 SCOD, 加快底物中多糖、蛋白质等物质的溶 解, 促进厌氧发酵的进程。因此可以说明, 固体酸预处理 
可以有效破坏木质素、纤维素以及半纤维素形成的网络结 构, 提高玉米芯与酶和降解微生物的接触面积, 从而促进 水解, 使SCOD浓度增加。

\subsection{2. 多糖浓度变化}

图5为预处理后发酵液中溶解性多糖的浓度变化情况。 固体酸和稀硫酸预处理组都表现出良好的促进水解效果, 并且都明显高于水热预处理组和对照组, 说明酸预处理能 够有效地破坏生物质的结构, 从而促进纤维素、半纤维素 水解产生可溶性糖类。通过曲线的变化情况, 可以看出实 验组多糖浓度在 0-5 d的变化较快, 反应比较剧烈, 表明反 应初期更适合微生物厌氧发酵。随着反应时间的延长, 各 个发酵系统的多糖浓度呈明显的下降趋势。这主要是因为 微生物利用可溶性多糖转化为VFAs的能力提高, 使多糖 转化速度大于生物质溶出多糖的速度, 总体表现为溶解性 多糖浓度逐渐趋向于零, 说明几乎所有的多糖都转化为 VFAs[19]。

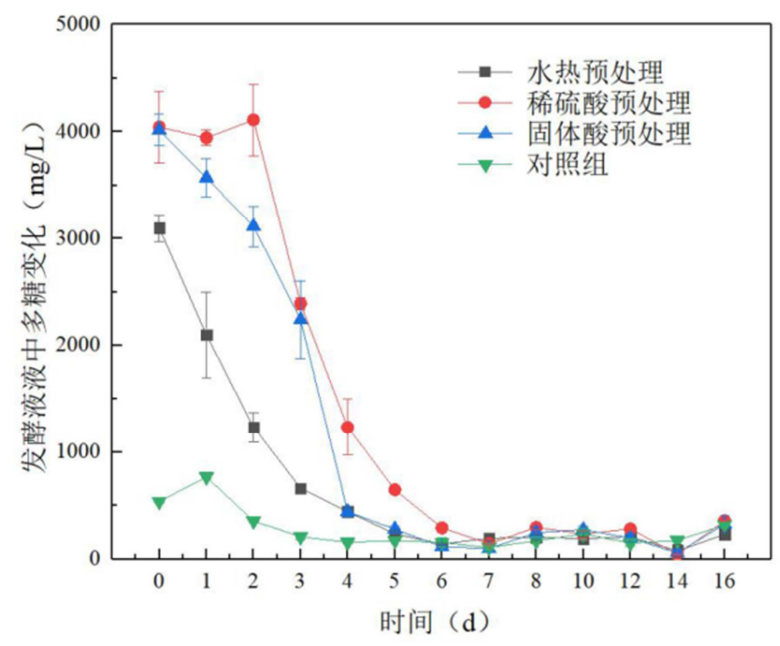

图5 发酵液中多糖浓度的变化。

\subsection{3. 蛋白质浓度变化}

图6为预处理后发酵液中溶解性蛋白质的浓度变化情 况。由图可知, 不同实验组发酵系统中初始蛋白质浓度不 同。水热预处理组溶出的蛋白质浓度最高, 这是由于水热 促进了蛋白质的溶出。固体酸和稀硫酸预处理组发酵液中 蛋白质浓度相近, 且低于水热预处理组, 可能是由于两个 发酵反应中水解作用过度, 破坏了蛋白质的结构, 并且蛋 白质结构中含有易被酸破坏的化学键, 这也会对蛋白质的 活性有很大影响[20]。除此之外, 导致此结果的原因也可 能是部分可溶性蛋白质被重新絮凝[19]。随着厌氧发酵反 应的进行, 各实验组发酵液中溶解性蛋白质浓度的变化趋 势相似, 都是先增大后减小。在厌氧发酵前期蛋白质浓度 增加是因为微生物消耗蛋白质的速度小于蛋白质溶出的 速度; 在厌氧发酵后期蛋白质浓度降低, 主要是因为随着 反应的进行, 厌氧微生物数量增多, 活性和总体代谢强度 增强, 使利用蛋白质转化为VFAs的速度大于蛋白质的产 生速度, 这与前文提到的VFAs产量增长情况一致。

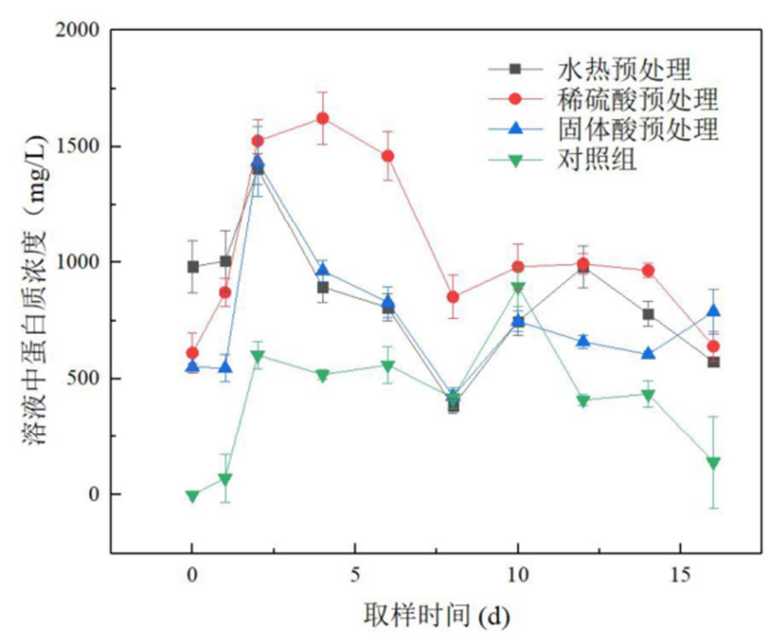

图6 发酵液中蛋白质浓度的变化。

\section{4. 结论}

本研究针对生物质结构复杂难以水解的问题, 开发了 固体酸预处理方法, 并比较固体酸、稀硫酸和水热预处理 促进玉米芯厌氧发酵产VFAs的效果, 结果表明, 预处理 后玉米芯厌氧发酵量显著提高, 固体酸预处理组发酵液中 VFAs浓度最高, 达到 $4055.15 \mathrm{mg} / \mathrm{L}$, 比对照组提高 $41.34 \%$, 并且显著高于其他实验组。固体酸预处理有效地破坏了玉 米芯的结构, 促进了玉米芯的水解, 提高了VFAs的产量。 实验完成后固体酸可回收利用, 但其重复使用后的催化性 能还需进一步分析, 并且固体酸失活后能使其重新活化的 方法也需进一步探究。

\section{参考文献}

[1] Bandara, Y.W., Gamage, P., Gunarathne, D.S. Hot water washing of rice husk for ash removal: The effect of washing temperature, washing time and particle size $[\mathrm{J}]$. Renewable Energy, 2020, 153:646-652.

[2] Zhou, A., Du, J., Varrone, C., Wang, Y., Wang, A., Liu, W. 2 VFAs bioproduction from waste activated sludge by coupling pretreatments with Agaricus bisporus substrates conditioning[J]. Process Biochemistry, 2014, 49(2):283-289.

[3] 何文修, 张智亮, 计建炳. 稻壳生物质资源利用技术研究 进展 [J]. 化工进展, 2016, 35(05).

[4] 徐超,阎宏,闻岳,周琪. 碱性条件促进植物生物质厌氧水解 及发酵产酸的研究 [J]. 安徽农业科 学,2011,39(36):22518-22520.

[5] 汪楚乔. 农村废弃生物质太阳能辅热中温两相厌氧产沼技 术研究[D]. 东南大学, 2017 .

[6] Wang, H.-Y., Zhang, C.-B., He, H., Wang, L. Preparation o fMagnetic Sulfonated Carbon-Based Solid Acid Catalysts for the Hydrolysis of Cellulose[J]. Acta Physico-Chimica Sinica, 2010:26. 
[7] Bureros, G.M.A., Tanjay, A.A., Cuizon, D.E.S., Go, A.W., Cabatingan, L.K., Agapay, R.C., Ju, Y.-H. Cacao shell-derived solid acid catalyst for esterification of oleic acid with methanol[J]. Renewable Energy, 2019, 138:489-501.

[8] Flores, K.P., Omega, J.L.O., Cabatingan, L.K., Go, A.W., Agapay, R.C., Ju, Y.-H. Simultaneously carbonized and sulfonated sugarcane bagasse as solid acid catalyst for the esterification of oleic acid with methanol[J]. Renewable Energy, 2019, 130:510-523.

[9] Chen, N., Zhang, G., Zhang, P., Tao, X., Wu, Y., Wang, S., Nabi, M. Rice husk-based solid acid for efficient hydrolysis and saccharification of corncob[J]. Bioresource Technology, 2019, 292:121915.

[10] Wang, S., Tao, X., Zhang, G., Zhang, P., Wang, H., Ye, J., Li, F., Zhang, Q., Nabi, M. Benefit of solid-liquid separation on volatile fatty acid production from grass clipping with ultrasound-calcium hydroxide pretreatment $[\mathrm{J}]$. Bioresource Technology, 2019, 274:97-104.

[11] Fang W., Zhang P., Zhang T., Requeson D.C., Poser M. Upgrading volatile fatty acids production through anaerobic co-fermentation of mushroom residue and sewage sludge: Performance evaluation and kinetic analysis[J]. Journal of Environmental Management, 2019, 241:612-618.

[12] Wainaina, S., Lukitawesa, Kumar Awasthi, M., Taherzadeh, M.J. Bioengineering of anaerobic digestion for volatile fatty acids, hydrogen or methane production: A critical review[J]. Bioengineered, 2019, 10(1):437-458.
[13] Lee W.S., Chua A.S.M., Yeoh H.K., Ngoh G.C. A review of the production and applications of waste-derived volatile fatty acids[J]. Chemical Engineering Journal, 2014, 235:83-99.

[14] Liu H., Shi J., Zhan X., Zhang L., Fu B., Liu H. Selective acetate production with $\mathrm{CO} 2$ sequestration through acetogen-enriched sludge inoculums in anaerobic digestion[J]. Biochemical Engineering Journal, 2017, 121:163-170.

[15] Xu, Z., Jiang, L. Butyric acid. Compr. Biotechnology, 2011, 207-215.

[16] Bhatia S.K., Yang Y.-H. Microbial production of volatile fatty acids: current status and future perspectives[J]. Reviews in Environmental Science \& Bio/technology, 2017, 16(2):327-345.

[17] Chen, Y., Jiang, S., Yuan, H., Zhou, Q., Gu, G. Hydrolysis and acidification of waste activated sludge at different $\mathrm{pHs}[\mathrm{J}]$. Water Research, 2007, 41(3):683-689.

[18] Rajinikanth R., Fabrice B. Anaerobic hydrolysis and acidification of organic substrates: Determination of anaerobic hydrolytic potential[J]. Bioresource Technology, 2011, 102:5653-5658

[19] Kang X.R., Zhang G.M., Chen L., Dong W., Tian W. Effect of initial $\mathrm{pH}$ adjustment on hydrolysis and acidification of sludge by ultrasonic pretreatment[J]. Industrial \& Engineering Chemistry Research, 2011, 50(22):12372-12378.

[20] 冯小黎,金业涛,苏志国. 分离纯化中蛋白质的不稳定性及 其对策[J]. 生物工程进展,2000,20(03):67-71. 